\title{
BERNSTEIN-DOETSCH TYPE RESULTS FOR QUASICONVEX FUNCTIONS
}

\author{
AtTila GilánYi, KaZimierZ NikOdem AND Zsolt PÁLES
}

\begin{abstract}
In this paper various quasiconvexity notions are considered and compared. The main goal is to show that, under the assumption of upper semicontinuity, Jensen-type quasiconvexity properties are equivalent to the corresponding ordinary quasiconvexity property. The results thus obtained are analogous to the classical theorem of Bernstein and Doetsch for convex functions.

Finally, the connection between approximate Jensen quasiconvexity and approximate quasiconvexity is investigated.
\end{abstract}

Mathematics subject classification (2000): 26B25, 39B62.

Key words and phrases: quasiconvex function, Jensen-quasiconvex function, $\varepsilon$-Jensen-quasiconvex function, upper semicontinuity.

\section{REFERENCES}

[1] F. Bernstein AND G. Doetsch, Zur Theorie der konvexen Funktionen, Math. Annalen 76 (1915), 514-526.

[2] H. J. GREENBERG AND W. P. PiersKalla, A review of quasiconvex functions, Operations Res. 19 (1971), $1553-1570$.

[3] D. H. HYERS, On the stability of the linear functional equation, Proc. Natl. Acad. Sci. U.S.A. 27 (1941), 222-224.

[4] D. H. Hyers And S. M. Ulam, Approximately convex functions, Proc. Amer. Math. Soc. 3 (1952), 821-828.

[5] J. L. W. V. JENSEN, Sur les fonctions convexes et les inéqualités entre les valeurs moyennes, Acta Math. 30 (1906), 179-173.

[6] C. T. Ng AND K. NiKodem, On approximately convex functions, Proc. Amer. Math. Soc. 118 (1993), $103-108$.

[7] K. Nikodem, Approximately quasiconvex functions, C. R. Math. Rep. Acad. Sci. Canada 10 (1988), 227-231.

[8] Zs. PÁLES, Bernstein-Doetsch-type results for general functional inequalities, Rocznik Nauk.-Dydakt. Prace Mat. 17 (2000), 197-206, Dedicated to Professor Zenon Moszner on his 70th birthday.

[9] A. W. Roberts and D. E. Varberg, Convex Functions, Academic Press, New York-London, 1973. 